\title{
The cytotoxicity of organobismuth compounds with certain molecular structures can be diminished by replacing the bismuth atom with an antimony atom in the molecules
}

\author{
Kumiko Kohri', Eiko Yoshida', Shuji Yasuike ${ }^{2,3}$, Tomoya Fujie', \\ Chika Yamamoto ${ }^{3,4}$ and Toshiyuki Kaji1,3
}

\begin{abstract}
${ }^{1}$ Faculty of Pharmaceutical Sciences, Tokyo University of Science, 2641 Yamazaki, Noda 278-8510, Japan ${ }^{2}$ School of Pharmacy, Aichi Gakuin University, 1-100 Kusumoto-cho, Chikusa-ku, Nagoya 464-8650, Japan ${ }^{3}$ Division of Bio-organometallics, Research Institute for Science and Technology, Tokyo University of Science, 2641 Yamazaki, Noda 278-8510, Japan

${ }^{4}$ Faculty of Pharmaceutical Sciences, Toho University, 2-2-1 Miyama, Funabashi 274-8510, Japan
\end{abstract}

[Contributed by Toshiyuki Kaji]

(Received February 19, 2015; Accepted February 19, 2015)

\begin{abstract}
Organic-inorganic hybrid molecules, which are composed of an organic structure and metal(s), are indispensable for synthetic chemical reactions; however, their toxicity has been incompletely understood. In the present study, we discovered two cytotoxic organobismuth compounds whose cytotoxicity diminished upon replacement of the intramolecular bismuth atom with an antimony atom. The intracellular accumulation of the organobismuth compounds was much higher than that of the organoantimony compounds with the corresponding organic structures. We also showed that both the organic structure and bismuth atom are required for certain organobismuth compounds to exert their cytotoxic effect, suggesting that the cytotoxicity of such a compound is a result of an interaction between the organic structure and the bismuth atom. The present data suggest that organobismuth compounds with certain molecular structures exhibit cytotoxicity via an interaction between the molecular structure and the bismuth atom, and this cytotoxicity can be diminished by replacing the bismuth atom with an antimony atom, resulting in lower intracellular accumulation.
\end{abstract}

Key words: Organobismuth, Organoantimony, Bio-organometallics, Cytotoxicity

\section{INTRODUCTION}

Organic-inorganic hybrid molecules are composed of an organic structure and metal(s). Since the early work of pioneers such as Grignard and Wittig in using hybrid molecules as reagents for chemical synthetic reactions (Grignard, 1900; Wittig and Schöllkopf, 1954), these molecules have become indispensable for synthetic chemistry. However, the effects of incorporation of metal(s) into organic structures on biological systems have not been completely understood. An expansion of the biological applications of organic-inorganic hybrid molecules (bioorganometallics) is expected.

The toxicity of organic-inorganic hybrid molecules appears to depend on the interaction between the molecular structure and the intramolecular metal(s) as well as the intrinsic cytotoxicity of either the molecular structure or the metal(s). However, there have not been adequate model compounds to examine this hypothesis.

We investigated the cytotoxicity of organobismuth compounds using a cell culture system and found that $\operatorname{tris}(N, N$-dimethylaminomethyl)phenylbismuthane exhibits selective cytotoxicity against vascular endothelial cells (Fujiwara et al., 2005). Recently, in our preliminary experiments, we found out that 2-( $N, N$-dimethylaminomethyl)phenylbis(4-methylphenyl)bismuthane (DAPBi) was cytotoxic to vascular endothelial cells, but the cytotoxicity disappeared when the bismuth atom was replaced with an antimony atom (the organoantimony compound is named DAPSb). In addition, a similar relationship between $\mathrm{Bi}$ phenyl- $N$-methyl-5,6,7,12-tetrahydrodibenz $[c, f][1,5]$ azabismocine (PMTABi) and $S b$-phenyl- $N$-methyl-5,6,7,12-

Correspondence: Toshiyuki Kaji (E-mail: t-kaji@rs.noda.tus.ac.jp) 
tetrahydrodibenz $[c, f][1,5]$ azastibocine (PMTAS) was observed in the cells.

In the present study, we investigated the relative cytotoxicity of DAPBi compared to DAPSb, and PMTABi compared to PMTAS in four cell types, including bovine aortic endothelial and smooth muscle cells, human fetal lung fibroblastic IMR-90 cells, and porcine kidney epithelial LLC-PK 1 cells. In addition, intracellular accumulation of the organobismuth and organoantimony compounds was measured to examine whether the difference in the cytotoxicity between these compounds is owing to differences in their intracellular accumulation.

\section{MATERIALS AND METHODS}

\section{Materials}

Arterial endothelial cells and smooth muscle cells derived from bovine aorta were purchased from DS Pharma Biomedical (Osaka, Japan). Fibroblastic IMR90 cells from human fetal lung and epithelial LLC-PK cells from porcine kidney were obtained from DS Pharma Biomedical. Dulbecco's modified Eagle's medium and calcium- and magnesium-free phosphate-buffered saline were obtained from Nissui Pharmaceutical (Tokyo, Japan). Fetal bovine serum was obtained from SigmaAldrich Chemical (St. Louis, MO, USA). Tissue culture dishes and plates were purchased from AGC Techno Glass (Chiba, Japan); 3,5-diaminobenzoic acid hydrochloride was acquired from Tokyo Kasei Industry (Tokyo, Japan), while the other reagents were obtained from Wako Pure Chemical Industries (Osaka, Japan). DAPBi, DAPSb, PMTABi, and PMTAS (Fig. 1) were synthesized as reported previously (Kawahara et al., 2011; Tokunaga et al., 2000; Ohkawa et al., 1989; Kakusawa and Kurita, 2006).

\section{Cell culture and treatments}

Vascular endothelial cells, vascular smooth muscle cells, IMR-90 cells, and LLC-PK 1 cells were cultured in 6 -well culture plates at $37^{\circ} \mathrm{C}$ in a humid atmosphere of $5 \% \mathrm{CO}_{2}$ in Dulbecco's modified Eagle's medium supplemented with $10 \%$ fetal bovine serum until confluent. The medium was discarded, and the cells were washed twice with Dulbecco's modified Eagle's medium supplemented with $10 \%$ fetal bovine serum. The cells were then treated with DAPBi, DAPSb, PMTABi, or PMTAS (at 0.5, 1 ,
[A]<smiles>Cc1ccc([Bi](c2ccc(C)cc2)c2ccccc2CN(C)C)cc1</smiles>

[C]<smiles>CCN(C)Cc1ccccc1[BH-](c1ccccc1)c1ccccc1</smiles>

[B]<smiles>Cc1ccc([Sb](c2ccc(C)cc2)c2ccccc2CN(C)C)cc1</smiles>

[D]<smiles>CN(Cc1ccccc1)Cc1ccccc1B(c1ccccc1)c1ccccc1</smiles>

Fig. 1. Structures of organobismuth and organoantimony compounds. [A]: 2-(N,N-dimethylaminomethyl)phenylbis(4-methylphenyl)bismuthane (DAPBi); [B]: 2-( $N, N$-dimethylaminomethyl)phenylbis(4-methylphenyl)stibane (DAPSb); [C]: $B i$-phenyl- $N$-methyl-5,6,7,12-tetrahydrodibenz[c,f][1,5] azabismocine (PMTABi); [D]: $S b$-phenyl- $N$-methyl-5,6,7,12tetrahydrodibenz $[c, f][1,5]$ azastibocine (PMTAS). 
Cytotoxicity of organobismuth and organoantimony compounds

2, and $5 \mu \mathrm{M}$ ) and incubated for $24 \mathrm{hr}$ in fresh Dulbecco's modified Eagle's medium supplemented with 10\% fetal bovine serum.

\section{Cytotoxicity and intracellular accumulation of the compounds}

After treatment, the cells were first observed microscopically to evaluate cell damage. Then, they were used for the determination of bismuth and antimony atoms as markers of intracellular accumulation using inductively coupled plasma mass spectrometry (ELAN DRC II; PerkinElmer, Waltham, MA, USA). The medium was discarded, and the cells were washed twice with ice-cold calcium- and magnesium-free phosphate-buffered saline and collected in $100 \mu \mathrm{L}$ of $0.5 \mathrm{M}$ Tris- $\mathrm{HCl}$ buffer solution (pH 6.8) containing 4\% sodium dodecyl sulfate and 20\% glycerol. The cells were then lysed by incubating at $95^{\circ} \mathrm{C}$ for $10 \mathrm{~min}$, and an aliquot of the lysate was incubated at $130^{\circ} \mathrm{C}$ for $48 \mathrm{hr}$ in $9.5 \mathrm{M}$ nitric acid and $7 \%$ hydrogen peroxide. The dried samples were dissolved in $2 \mathrm{~mL}$ of $0.1 \mathrm{M}$ nitric acid, and the content of bismuth and antimony atoms was analyzed. Another aliquot of the cell lysate was analyzed for DNA content by the fluorometric method (Kissane and Robins, 1958) to express the content of bismuth and antimony as pmol/ $\mu \mathrm{g}$ DNA.

\section{RESULTS}

Morphological observation (Fig. 2A) showed that DAPBi exhibited cytotoxicity in vascular endothelial and smooth muscle cells, fibroblastic IMR-90 cells, and epithelial LLC-PK 1 cells in a dose-dependent manner. In contrast, DAPSb did not show such cytotoxicity in these four cell types. DAPBi accumulated within these cell types, whereas the accumulation of DAPSb was very low in each cell type tested (Fig. 2B).

Figure 3 shows the morphological observation of the four cell types after exposure to PMTABi or PMTAS and the intracellular accumulation of the organometallic compounds; similar to our previous observations, the organobismuth compound, PMTABi, accumulated extensively within the cells and exhibited cytotoxicity, whereas the organoantimony compound, PMTAS, accumulated to a lesser extent and demonstrated no cytotoxicity.

In order to examine whether the cytotoxicity of organobismuth compounds depends on the organic structure, bismuth atom, or interaction between the molecular structure and bismuth atom, the cytotoxicity of PMTABi was compared to dibenzylmethylamine, a compound in which the bridge containing the bismuth atom was removed, triphenyl bismuth, a compound in which the bridge containing the nitrogen atom was eliminated, and bismuth nitrate, an inorganic bismuth (Fig. 4). Among the compounds tested, only PMTABi exhibited cytotoxicity, suggesting that the cytotoxicity of the organobismuth compound requires the complete structure of the molecule.

\section{DISCUSSION}

Although the toxicity of organic and inorganic compounds has been well investigated, the toxicity of organic-inorganic hybrid molecules has not been completely understood. In general, the toxicity of the hybrid molecules was believed to depend on the metal(s) rather than the organic molecular structure, even though this assertion is without reliable evidence. In spite of the knowledge that the cytotoxicity of inorganic bismuth compounds is low (Serfontein and Mekel, 1979) while that of inorganic antimony is high (Mann et al., 2006), the present data demonstrated that there are cases where organobismuth compounds are cytotoxic but organoantimony compounds with the same organic structure are nontoxic. This suggests that the cytotoxicity of hybrid molecules does not necessarily depend on either the organic structure or metal atom(s) in the molecules.

The mechanisms underlying the toxicity of hybrid molecules appear to be complex. We have shown that PMTA$\mathrm{Bi}$ is toxic to vascular endothelial cells, but the structural components of the organobismuth compound by themselves are nontoxic, suggesting that the interaction of bismuth with the organic structure is important for the cytotoxicity of PMTABi. It is most likely that the difference in cytotoxicity between organic and inorganic bismuth compounds, and among the various organobismuth compounds, is based on this interaction. Not only chemical and biological approaches, but also physical approaches such as electronic state analysis may be required for understanding this interaction.

The cytotoxicity of DAPBi and PMTABi was much stronger than that of DAPSb and PMTABi, respectively, in all cell types tested, suggesting that the cytotoxicity of organobismuth compounds is independent of cell type and can be diminished by replacing the bismuth atom with an antimony atom. There may be common mechanisms underlying the cytotoxicity of organobismuth compounds. On the other hand, DAPBi and PMTABi accumulated within the cells whereas the accumulation of DAPSb and PMTABi was very less, suggesting that there may be a transport system for organobismuth compounds, which cannot or very slightly, transport organoantimony compounds into the cells. Recently, transporters for divalent cations, including zinc, copper, manganese, iron, and 
[A] Vascular endothelial cells
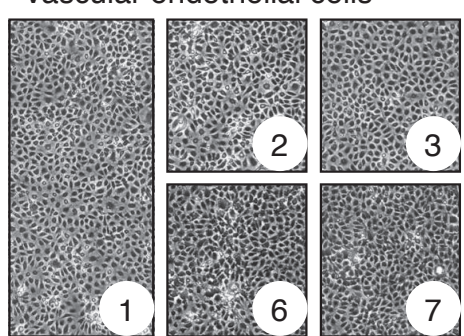

Fibroblastic IMR-90 cells
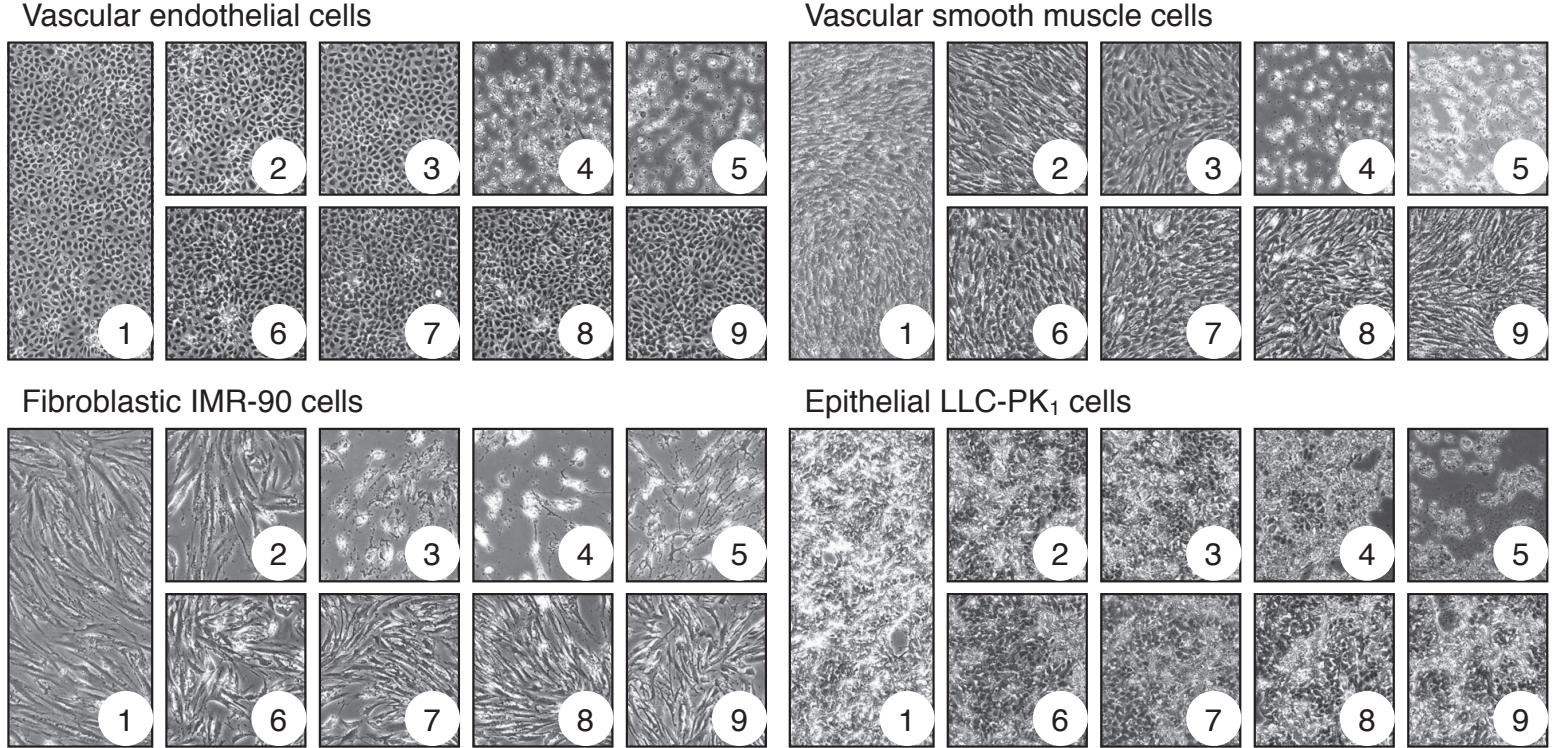

Epithelial LLC-PK 1 cells
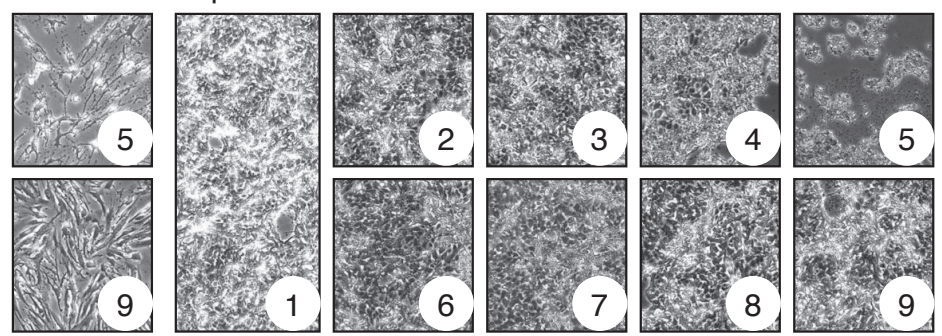

[B] Vascular endothelial cells

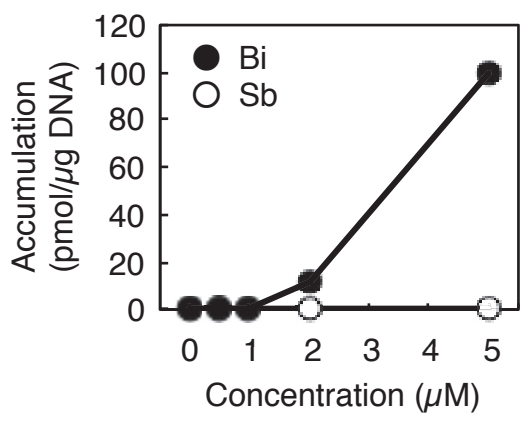

Fibroblastic IMR-90 cells

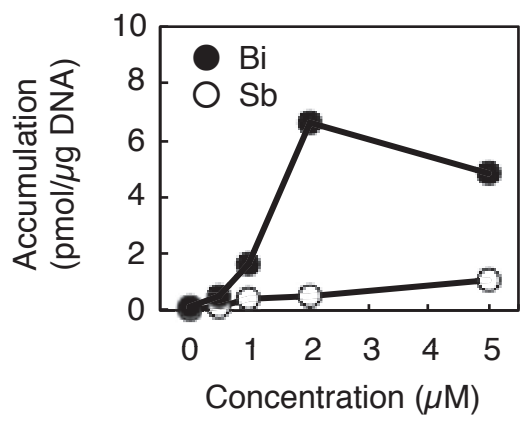

Vascular smooth muscle cells

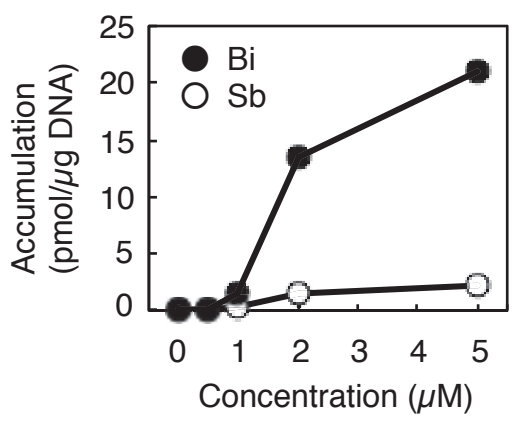

Epithelial LLC-PK 1 cells

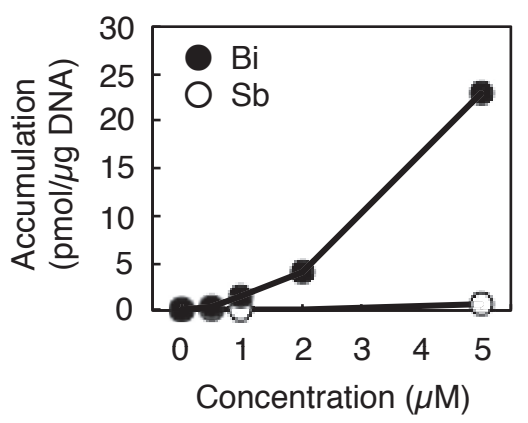

Fig. 2. Cytotoxicity and intracellular accumulation of DAPBi and DAPSb in vascular endothelial cells, vascular smooth muscle cells, fibroblastic IMR-90 cells, and epithelial LLC-PK 1 cells. Bovine aortic endothelial and smooth muscle cells, human fetal lung fibroblastic IMR-90 cells, and porcine kidney epithelial LLC-PK, cells were treated with DAPBi or DAPSb $(0.5,1,2$, and $5 \mu \mathrm{M})$ for $24 \mathrm{hr}$. [A]: Morphological appearance after treatment with DAPBi or DAPSb. 1: Control; 2: DAPBi $(0.5 \mu \mathrm{M})$; 3: DAPBi $(1 \mu \mathrm{M})$; 4: DAPBi $(2 \mu \mathrm{M})$; 5: DAPBi $(5 \mu \mathrm{M})$; 6: DAPSb $(0.5 \mu \mathrm{M})$; 7: DAPSb $(1 \mu \mathrm{M})$; 8: DAPSb $(2 \mu \mathrm{M})$; 9: DAPSb $(5 \mu \mathrm{M})$. [B]: Intracellular accumulation of DAPBi and DAPSb. $\bullet$, DAPBi; $\circ$, DAPSb. 
Cytotoxicity of organobismuth and organoantimony compounds

[A] Vascular endothelial cells
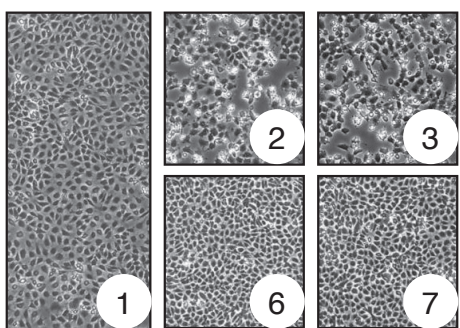

Fibroblastic IMR-90 cells
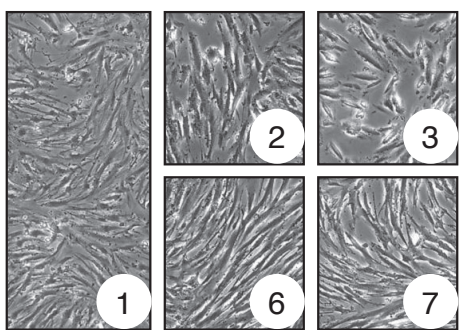

[B] Vascular endothelial cells

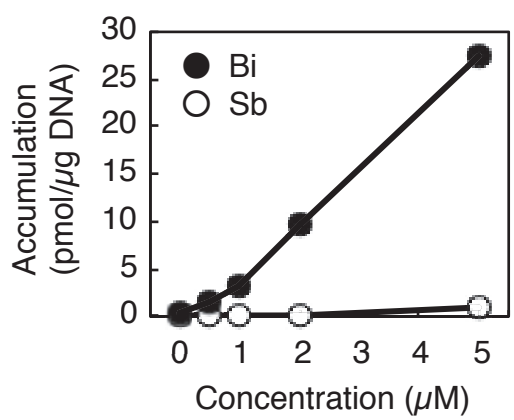

Fibroblastic IMR-90 cells

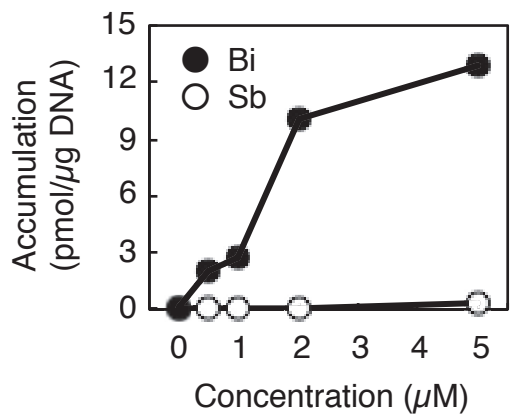

Vascular smooth muscle cells
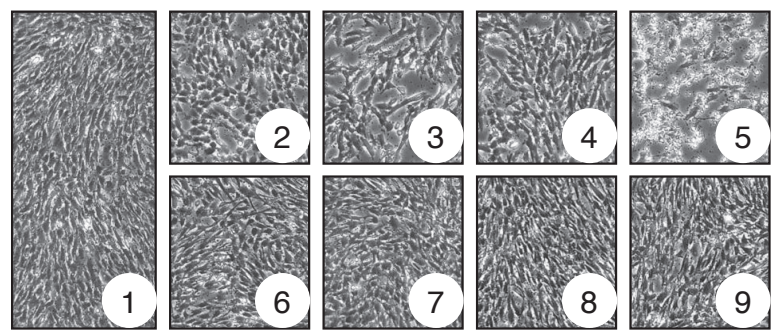

Epithelial LLC-PK ${ }_{1}$ cells
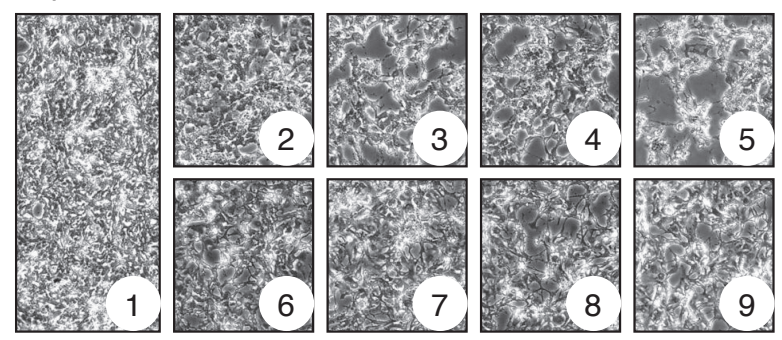

Vascular smooth muscle cells

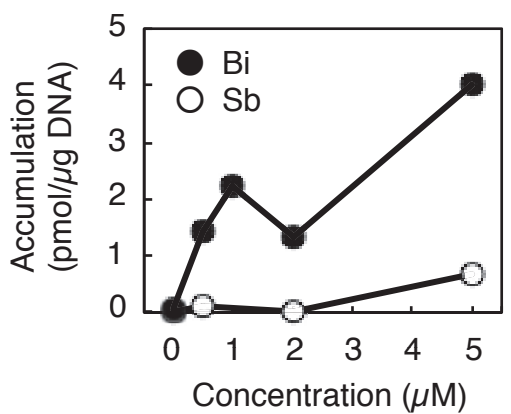

Epithelial LLC-PK ${ }_{1}$ cells

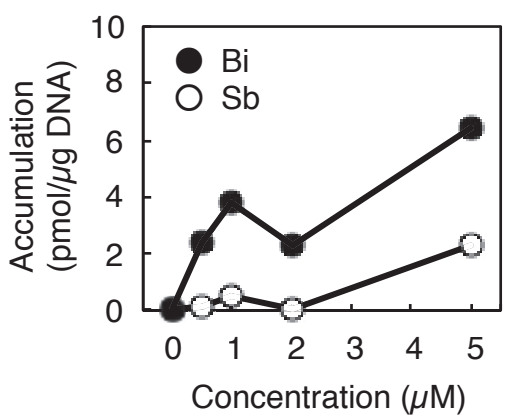

Fig. 3. Cytotoxicity and intracellular accumulation of PMTABi and PMTAS in vascular endothelial cells, vascular smooth muscle cells, fibroblastic IMR-90 cells, and epithelial LLC-PK, cells. Bovine aortic endothelial and smooth muscle cells, human fetal lung fibroblastic IMR-90 cells, and porcine kidney epithelial LLC-PK 1 cells were treated with PMTABi or PMTAS $(0.5,1,2$, and $5 \mu \mathrm{M})$ for $24 \mathrm{hr}$. [A]: Morphological appearance after treatment with PMTABi or PMTAS. 1: Control; 2: PMTABi $(0.5 \mu \mathrm{M})$; 3: PMTABi $(1 \mu \mathrm{M})$; 4: PMTABi $(2 \mu \mathrm{M})$; 5: PMTABi $(5 \mu \mathrm{M})$; 6: PMTAS $(0.5 \mu \mathrm{M})$; 7 : PMTAS $(1 \mu \mathrm{M})$; 8: PMTAS $(2 \mu \mathrm{M})$; 9: PMTAS $(5 \mu \mathrm{M})$. [B]: Intracellular accumulation of DAPBi and DAPSb. $\bullet$, PMTABi; $\circ$, PMTAS. 


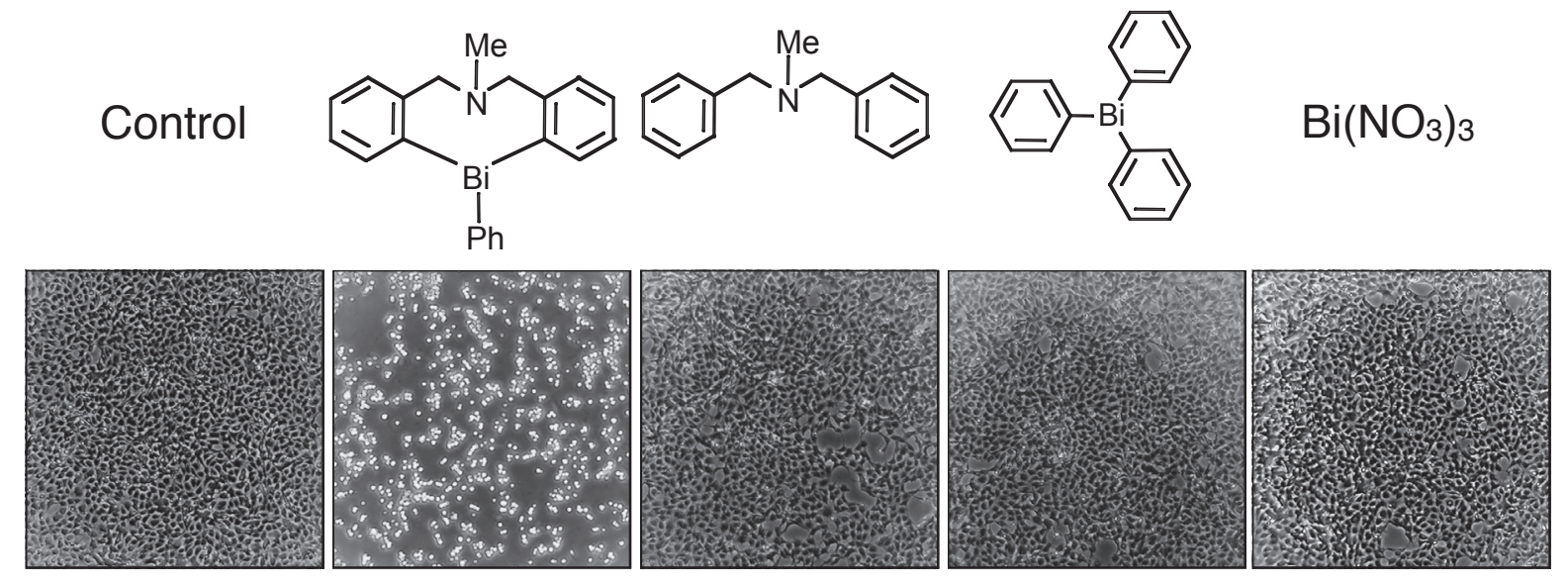

Fig. 4. Cytotoxicity of PMTABi, dibenzylmethylamine, triphenyl bismuth, and bismuth nitrate in vascular endothelial cells. Bovine aortic endothelial cells were treated with PMTABi, dibenzylmethylamine, triphenyl bismuth, or bismuth nitrate $(10 \mu \mathrm{M}$ each) for $24 \mathrm{hr}$.

cadmium have been identified (Gaither and Eide, 2001; Mackenzie et al., 2007; Kambe, 2011) however, it is unlikely that there are selective transporters for organobismuth compounds. A biomolecule internalization system that has a high affinity for organobismuth compounds with a certain molecular structure may be involved in the transport of organobismuth compounds. Replacement of the bismuth atom with an antimony atom may result in lowering this affinity.

The present study elucidated several aspects of organobismuth compounds' cytotoxicity. Organobismuth compounds with certain molecular structures were shown to be cytotoxic, and this cytotoxicity can be diminished by replacing the bismuth atom with an antimony atom. This phenomenon was observed in various cell types including vascular endothelial and smooth muscle cells, fibroblastic cells, and epithelial cells. The intracellular accumulation of organobismuth compounds with certain molecular structures is high whereas that of organoantimony compounds with the corresponding organic structures is very low; this difference appears to be one of the mechanisms underlying the stronger cytotoxicity of the organobismuth compounds compared to the organoantimony compounds. Finally, we showed that the complete structure is required for organobismuth compounds to exhibit cytotoxicity; the interaction between the organic structure and the bismuth atom may be important for cytotoxicity. Although it is postulated that the cytotoxicity of hybrid molecules is dependent on the intramolecular metal(s), organic structure, or interaction between the metal(s) and the structure, further studies will be required to fully understand the toxicity of organic-inorganic hybrid molecules.

\section{ACKNOWLEDGMENTS}

This work was supported by a Grant-in-Aid for Challenging Exploratory Research \#24659058 (to T. K.) and a Grant-in-Aid for Scientific Research (C) \#23590156 (to C. Y.) from the Japan Society for the Promotion of Science.

Conflict of interest---- The authors declare that there is no conflict of interest.

\section{REFERENCES}

Fujiwara, Y., Mitani, M., Yasuike, S., Kurita, J. and Kaji, T. (2005): An organobismuth compound that exhibits selective cytotoxicity to vascular endothelial cells in vitro. J. Health Sci., 51, 333-340.

Grignard, V. (1900): Some new organometallic combinations of magnesium and their application to the synthesis of alcohols and hydrocarbons. C. R. Acad. Sci., 130, 1322-1324.

Gaither, L.A. and Eide, D.J. (2001): Eukaryotic zinc transporters and their regulation. Biometals, 14, 251-270.

Kambe, T. (2011): An overview of a wide range of functions of $\mathrm{ZnT}$ and Zip zinc transporters in the secretory pathway. Biosci. Biotechnol. Biochem., 75, 1036-1043.

Kakusawa, N. and Kurita, J. (2006): Hypervalent organoantimony compound 12-aryl-tetrahydrodibenz $[\mathrm{c}, \mathrm{f}][1,5]$ azastibocine: New transmetallating agent for palladium-catalyzed arylation of organic halides. Heterocycles, 68, 1335-1348.

Kawahata, M., Yasuike, S., Kinebuchi, I., Yamaguchi, K. and Kurita, J. (2011): \{2-[(Dimethylamino)methyl]phenyl $\}$ bis(4- 
Cytotoxicity of organobismuth and organoantimony compounds

methylphenyl)bismuthane. Acta Cryst. E, 67, m25.

Kissane, J.M. and Robins, E. (1958): The fluorometric measurement of deoxyribonucleic acid in animal tissues with special reference to the central nervous system. J. Biol. Chem., 233, 184-188.

Mackenzie, B., Takanaga, H., Hubert, N., Rolfs, A. and Hediger, M.A. (2007): Functional properties of multiple isoforms of human divalent metal-ion transporter 1 (DMT1). Biochem. J., 403, 59-69.

Mann, K.K., Davison, K., Colombo, M., Colosimo, A.L., Diaz, Z., Padovani, A.M., Guo, Q., Scrivens, P.J., Goa, W., Mader, S. and Miller, W.H.Jr. (2006): Antimony trioxide-induced apoptosis is dependent on SEK1/JNK signaling. Toxicol. Lett., 160, 158170.

Ohkata, K., Takemoto, S., Ohnishi, M. and Akiba, K. (1989): Synthesis and chemical behaviors of 12 -substituted dibenz $[\mathrm{c}, \mathrm{f}][1,5]$ azastibocine and dibenz [c,f][1,5]azabismocine derivatives: evidences of 10-Pn-4 type hypervalent interaction. Tetrahedron Lett., 30, 4841-4844.

Serfontein, W.J. and Mekel, R. (1979): Bismuth toxicity in man II. Review of bismuth blood and urine levels in patients after administration of therapeutic bismuth formulations in relation to the problem of bismuth toxicity in man. Res. Commun. Chem. Pathol. Pharmacol., 26, 391-411.

Tokunaga, T., Seki, H., Yasuike, S., Ikoma, M., Kurita, J. and Yamaguchi, K. (2000): Confirmation and characterization of the hypervalent $\mathrm{Sb} \cdots \mathrm{N}$ bonding recognized in triarylstibanes bearing an amino side chain by X-ray, NMR and theoretical calculations. Tetrahedron, 56, 8833-8839.

Wittig, G. and Schöllkopf, U. (1954): Triphenylphosphinemethylene as an olefin-forming reagent. Chem. Ber., 97, 1318-1330. 